\title{
Educación, diferencias subjetivas y culturales. ¿De la multiculturalidad a la interculturalidad?
}

DOI: https://doi.org/10.32870/dse.v0i8.303

\author{
Emma Ruiz Martín del Campo*
}

Ser otro no significa ser radicalmente distinto para el prójimo. Por el contrario, yo creo que las relaciones más profundas surgen entre dos personas que aceptan su diferencia. Si no existe tal diferencia, no hay verdadero diálogo.

Edmond Jabès

Resumen: El inusitado incremento de las migraciones en la llamada 'posmodernidad', ha dado lugar a sociedades en las que conviven sujetos de múltiples culturas. Los adultos que emigran con la esperanza de encontrar mejores condiciones de vida, llevan muchas veces con ellos a hijos que, estando en edad de formación, son aceptados en escuelas del país receptor. En las clases en las que un maestro tiene que interactuar con alumnos de, en ocasiones hasta 15 culturas diferentes, surge una atmósfera que ha dado pie a las reflexiones planteadas en este artículo: ¿Qué les es dado hacer a los educadores (en sentido amplio) que tienen que enfrentar tal gama de diferencias subjetivas y culturales y además cumplir su tarea de gestar conocimiento? ¿Es posible pasar de la multiculturalidad a la interculturalidad? A fin de dar pautas de posibles respuestas para tales cuestiones, se revisan las concepciones de cultura de teóricos como Mario Erdheim y Homi Bhabha y las aportaciones que para entender la subjetividad ha hecho el psicoanálisis, desde el mismo Freud hasta quienes se preguntan por las formas en que estamos "sujetos a nuestro propio y múltiple ser", como Anthony Elliot. Palabras clave: educación, sujetos, cultura, multiculturalidad, interculturalidad.

\begin{abstract}
The exorbitant increase of migrations in the so-called 'post-modernity' has produced societies in which subjects proceeding from several cultures live. Adults that migrate with the hope of finding a better way of life often take their children with them. These children then get a place at a school in the country that has welcomed them. In classes in which a teacher must sometimes work with as many as fifteen different cultures, there is an atmosphere that has made me reflect about the questions formulated in this article: What can education- professionals do when they have to teach and create knowledge while facing so many subjective and cultural differences? Is it possible to evolve from a multicultural to an intercultural atmosphere? In order to get some possible answers to such questions, I go through culture conceptions of theoretical intellectuals like Mario Erdheim and Homi Bhabha and through proposals about ways of understanding subjectivity, made from psychoanalysts from Freud himself to others like Anthony Elliot, who reflect about how we are subject to our own and complex being. Key words: education, subjects, culture, multiculturalism, interculturalism.
\end{abstract}

*Doctora en Ciencias Sociales por la Universidad. Goethe de Frankfurt. Psicoanalista en práctica privada. Miembro del SNI nivel 2. Profesora-investigadora Titular "C" en el Departamento de Estudios en Educación, Universidad de Guadalajara. Correo electrónico: emmaruiz0808@hotmail.com 


\section{Antecedentes}

Del verano de 1998 al de 1999 fui docente invitada en el Instituto de Ciencias de la Educación de la Universidad Philipps de Marburg, Alemania. Uno de los cursos que impartí fue La comprensión etnopsicoanalítica de lo extranjero. Algunos maestros de 'Gymnasium', periodo que corresponde a la escolaridad mexicana que va del $5^{\circ}$ grado de primaria al bachillerato, me pidieron la posibilidad de participar como oyentes en la clase. No tuve inconveniente alguno en que se integraran, pero sí les pedí que no fueran textuales en cuanto a jugar el papel de oyentes. Excepto porque no requerían una nota final, yo los consideraría -les expliqué- participantes con pleno derecho en las discusiones grupales. En la primera clase pedí a todos que se presentaran brevemente ante el grupo, añadiendo a sus datos personales la exposición del motivo por el que estaban interesados en el tema de lo extranjero. Quienes trabajaban frente a grupos explicaron que año con año crecía el número de alumnos extranjeros que tenían en su clase, pero que además también aumentaba el número de culturas de procedencia de dichos alumnos, llegando a ser en ocasiones hasta 15 las reunidas en el aula. Ante el reto que eso implicaba querían -argumentaron- entender cuanto fuera posible de lo que acontece en el encuentro con los otros, los diferentes. Esto ocurría hace más de 15 años, a partir de entonces ha habido cambios en el mundo que han aumentado las migraciones y no han cesado los conflictos entre etnias y culturas diferentes. Desde el $1^{\circ}$ de enero del presente año, por ejemplo, Alemania concede oficialmente permiso de trabajo a ciudadanos búlgaros y rumanos, y muchos de ellos no llegan solos, traen consigo a niños que son inscritos en la escuela para su educación formal. En el ámbito de la multiculturalidad y el potencial de conflicto que encierra, piénsese en lo que ocurre hoy en día: 2 marzo del 2014 entre rusos y ucranianos en torno a Crimea, territorio históricamente en disputa, donde hay una mayor parte de la población ligada a la cultura rusa y otra numéricamente inferior, a la ucraniana. El conflicto amenaza con llegar al terreno de la lucha armada. (Las implicaciones y repercusiones internacionales de este conflicto son un tema por demás importante, pero quedan fuera del ámbito de reflexión de este trabajo).

La multiculturalidad y la importancia de la gesta de interculturalidad son cuestiones más vigentes que nunca en nuestro mundo cada vez más globalizado. De ahí mi decisión de hacer una aportación al debate contemporáneo, rastreando aportaciones al tema tanto desde los desarrollos teóricos, como desde el ámbito de lo artístico y desde el saber de la experiencia.

La multiculturalidad es un fenómeno de crecientes dimensiones en el siglo XXI, que definiremos, para los efectos de este trabajo, como la existencia de sujetos procedentes de grupos culturales diferentes en un contexto obligadamente compartido, sin que se den todavía entre ellos cruces simbólicos posibilitados por la comunicación verbal y/o no verbal, las identificaciones, el intercambio emocional y las transacciones indispensables para enfrentar el reto de tareas y/o actividades lúdicas compartidas.

El encuentro entre los sujetos con una impronta cultural diferente, que comparten en un primer momento el multiculturalismo de la diversidad, puede dar lugar a procesos de hibridación. Expli- 
Educación, diferencias subjetivas y culturales. ¿De la multiculturalidad a la interculturalidad?

quémonos: cuando los sujetos a pesar de sus diferencias culturales o en base a las mismas, conscientes de ser todos extranjeros ante el otro, se respetan recíprocamente y se aceptan como semejantes; esto es, marcados todos por la condición de humanos, necesitados de aceptación y resguardo para sobrevivir, mortales capaces de tomar consciencia de serlo, puede darse la apertura necesaria que mueva al esfuerzo por traducir lo que los diferentes expresan (así sea en un principio a partir de objetos, lenguaje corporal o artístico: música, pintura, etcétera). Con la apertura a los extranjeros crece el potencial de gestar nuevas producciones culturales que son una mezcla de las aportaciones de las distintas formas de experimentar y comprender el mundo, la vida, las relaciones.

La interculturalidad, que es otro de los términos alrededor de los cuales giran las reflexiones aquí presentadas, es la hibridez lograda; esto es, la inscripción y puesta en práctica de nuevas formas culturales producto de la articulación de las diferencias.

\section{"La clase"/"entre los muros": ¿la clase negadora de lo social que acuña a los sujetos?}

"La clase" es una película que recibió tal título en español y que se basa en la obra del escritor francés François Bégadeau Entre les murs ${ }^{1}$. Aborda la relación maestro-alumnos en una sociedad multicultural. Invita a reflexionar sobre la institución escuela, su diseño jerárquico y el proceso educativo como utopía, en el caso que se nos presenta fracasada, de un diálogo y una relación democrática entre seres humanos.

La película nos muestra al salón de clases como un ámbito más o menos desligado de interacciones directas con el contexto en que la escuela se ubica, como un apartado de una institución que separa de lo social externo, y que en momentos puede hacer pensar en un reformatorio o escuela disciplinaria.

El desarrollo de la trama puede llevar al espectador a preguntarse si esa educación, como ahí se hace ver, ¿es para la vida o contra la misma? ¿cómo pueden alumnos de tantas culturas diferentes asimilar los conocimientos que ahí se les proponen, que para muchos son por múltiples razones incomprensibles, y que de acuerdo a su cultura de socialización otros encuentran sinsentido, desligados de aplicaciones a la que hasta entonces había sido su experiencia, su haber de conocimientos aplicables a la solución de problemas cotidianos y a las relaciones en el que antaño fuera su contexto existencial? ¿cómo podrían, maestros insertados en una estructura escolar jerárquica, en la que la prioridad es hacer valer las concepciones francesas de educación, interactuar con alumnos de culturas muy diferentes, partiendo para el acto educativo de una profunda comprensión de las necesidades que ellos tienen como sujetos?

\footnotetext{
${ }^{1}$ Las traducciones del francés, del inglés y del alemán de citas incluidas en este trabajo son de mi autoría: Emma Ruiz.
} 
Laurent Cantent, el director de la película, premiada por triple partida ("Palme d'or: Cannes 2008, "Academy Award" y premio "Opening night" del festival de cine de Nueva York), nos presenta múltiples ejemplos de la sensación de absurdo que puede despertarse en adolescentes que, procedentes de realidades culturales diferentes, son aceptados en una escuela parisina y se adentran en un proceso que busca asimilarlos a las formas de vida francesas. Pero, podemos preguntarnos, ¿existe lo "francés"?, y si es así, ¿en qué consiste?

Al recibir la Palma de Oro en el Festival de Cannes en 2009, Cantet comentó: "La película que quisimos hacer habría de ser como la sociedad francesa - con muchos rostros, viva y compleja, con conflictos que no quisimos ocultar".

Tanto un salón de clases como un salón de maestros pueden estar al servicio de la educación, en el mejor sentido del término: facilitación de la reflexión y del reconocimiento de las emociones, promoción del encuentro respetuoso entre sujetos, gesta de conocimientos y tareas creativas susceptibles de integrarse en el quehacer cultural, búsqueda de consensos sobre normas, asunción de una ley que iguale a todos como semejantes, etcétera. Pero también pueden servir de ring para duelos de poder y enfrentamientos étnicos y culturales.

Destacaremos algunos párrafos del libro Entre les murs que dan cuenta de las dinámicas que pueden surgir en una clase multicultural en torno a las diferencias y las incomprensiones, en medio de las pretensiones de educación y de encuentro.

Dico confronta al profesor de francés, uno de los principales protagonistas de la obra, de esta forma:

D: -Señor, no quiero estar en esta clase, está totalmente podrida.

P: -¿Por qué está podrida?

D: -Incluyéndolo a usted, prof principal, no se hace nada.

P: -¡Apúrate! (Bégaudeau, 2007: 14).

Tras recibir la orden de esbozar por escrito un autorretrato en diez líneas pregunta Souleymane:

S: -¿Para qué hacemos esto?

P: -Les pido a todos mis grupos que lo hagan.

S: -No sirve para nada.

P: - Sirve para conocerte

S: -Pero de usted no se sabe nada.

P: - He escrito mi nombre en el pizarrón (ibidem: 18).

El profesor observa a un muchacho, al que se describe como pequeño y bronceado, sentado en la primera fila en el salón de clases y haciendo varios intentos de escribir. Logra decir su nombre 
Educación, diferencias subjetivas y culturales. ¿De la multiculturalidad a la interculturalidad?

leyéndolo de la hoja que les ha pedido poner doblada sobre su mesa y lo llama, pero Mezut parece no haberse dado por aludido, por lo que el profesor lo increpa:

P: - Mezut, es a mí al que se mira, ¿sí o no?

Dado que el chico murmura un 'sí' que el profesor califica de ‘sin convicción', le ordena:

P: - Vendrás a verme al final de la hora. Leemos luego la siguiente aclaración en Entre les murs: "El tim$b^{2}{ }^{2}$, ha tenido el efecto de un petardo lanzado dentro de una jaula de pájaros adormecidos. Miro de reojo a Mezut que se preguntaba si yo había olvidado o no ${ }^{3}$, pero prefiere no arriesgarse y se acerca en silencio, poniendo su autorretrato a un lado de mi lista de asistencias". Y luego continúa el diálogo:

P: - ¿Vas a ser así todo el año? Y el texto añade: "Su cabeza agachada ocultaba no sé qué gesto".

P: - Te escucho. ¿Vas a ser así todo el año?

M: - ¿Así cómo?

P: - Así dándote tono: 'me doy la vuelta sin detenerme o sonrío tontamente cuando me hablan'.

M: - Hay algo que yo no había entendido.

P: - Porque si eres así todo el año, va a ser la guerra y tú vas a perder. Si es la guerra, será una pesadilla para ti. Si haces las cosas bien, y esto va bien, tendremos un buen fin de jornada.

M: - Gracias. Hasta la vista, señor (ibídem: 19).

Transmito lo que se nos dice de la interacción entre Khoumba y el profesor, que dará luego pie a sustanciales reflexiones:

"El profesor de francés solicita a Khoumba la tarea que le ha pedido realizar como castigo por su negativa a leer un texto durante la clase (...) Khoumba se aproxima desde el fondo de la clase con una hoja que deja caer sobre el escritorio sin decir palabra. El escrito de Khoumba inicia con las siguientes consideraciones: 'Un adolescente aprende poco a poco a respetar a sus profesores a causa de las amenazas de éste que le despiertan el temor de tener problemas. Estos no son sino ejemplos. Yo lo respeto a usted y el respeto debe ser mutuo. Como por ejemplo yo no le digo que usted es histérico, entonces ¿por qué usted me lo dice? (...) Yo no sé por qué usted juega conmigo. A usted le corresponde enriquecer mis conocimientos de francés. Mi responsabilidad es de cumplir con todos los cursos a fondo, así ya no habrá conflictos 'para nada' si usted no me provoca. Tal vez he sido a veces insolente, pero si no me da motivo para ello, no lo soy. Bueno, vuelvo a la cuestión que nos ocupa. Cuando digo 'a causa de las amenazas de éste' me refiero, por ejemplo a lo que usted ha escrito en mi carnet: 'me veré obligado a tomar medidas más graves', pues bien, eso es una

\footnotetext{
${ }^{2}$ Que indica el fin de la clase.

${ }^{3}$ La petición de verlo al final de la clase.
} 


\section{Emma Ruiz Martín del Campo}

amenaza (según yo). Y cuando hablo de 'el temor de tener problemas' quiero decir que la persona teme ser enviada o reenviada a la oficina del director. Por mi parte, me comprometo a respetarlo si esto es mutuo ${ }^{4}$. De todas formas, yo no lo miraré más, a fin de que usted no diga que lo miro con insolencia. Y normalmente en un curso de francés uno debe hablar del francés y no de su abuela o su hermana. Es por esta razón que a partir de ahora yo no le hablaré más"5 (ibídem: 69-70).

El profesor comenta: "Yo había explicado el victimismo, y Mohammed-Ali había dicho que los árabes reconocían que ellos eran tan racistas como muchos, pero que había otros todavía peores: los de Martinica, que se creían más franceses que los árabes y Faiza había dicho que los martinicos se creían más franceses que los de Mali, y yo había dicho que no se debe generalizar", y tras este señalamiento varios alumnos intervienen:

A1: - Señor, el problema es que la naturaleza humana, el hombre querrá siempre destruir lo que no se le parece, eso es todo, así es, es fatal.

A2: - Lo que se necesitaría sería un enemigo común, con eso todo el mundo se reconciliaría. Basta con designarlo y ya está.

A3: - Además eso resolvería la sobrepoblación, parece que el problema es que hay demasiada gente (ibídem: 70/71).

A lo largo de la obra hay repetidas menciones a detalles disciplinarios considerados muy importantes en la escuela, entre ellos el pedir a los alumnos que se despojen de coberturas que para ellos son muchas veces insignias religiosas. La tendencia a desmarcar las diferencias toca la intimidad y las creencias de los sujetos, en última instancia lo que los hace ser quienes son:

"Souleymane entró a la clase con su capucha del Rabatt. Esperé a que se sentara y le indiqué:

P: - La capucha, Souleymane, por favor. Y también el bonete (ibídem: 75).

Las diferentes tradiciones, preferencias políticas y religiosas son tema de una reunión de los docentes con el director, en la que también participan los alumnos representantes de grupo.

"María abrió la sesión proponiendo la enmienda del reglamento acerca de los signos políticos y religiosos. Sandra ${ }^{6}$ (levantó la mano y preguntó por el sentido exacto de 'proselitismo'. De cuatro o cinco bocas marcadas por la solemnidad emergió el mismo número de definiciones distintas, en las que estaban implí-

\footnotetext{
${ }^{4}$ Negritas de la autora.

${ }^{5}$ Alusión a la petición de que hicieran su autorretrato.

${ }^{6}$ Una alumna que representaba a los estudiantes en el consejo escolar.
} 
Educación, diferencias subjetivas y culturales. ¿De la multiculturalidad a la interculturalidad?

citas cuestiones de tolerancia, de respeto, de valores comunes, de república. Considerando esta polifonía, el director propuso dejar el voto ${ }^{7}$ para el final de la sesión y abordar el segundo punto del orden del día" (ibídem: 76-77).

\section{Los sujetos de la educación}

El hecho educativo, como complejo fenómeno de socialización, alude al hombre (en el sentido genérico). Los humanos formamos parte de una especie que quedó despojada en el curso de la evolución de la mayor parte de conductas estereotipadas, pautadas por instintos y hubo de desarrollar cultura, transmisible en buena medida a través de educación, para sobrevivir.

María Moliner nos propone varias definiciones de educación. En la primera se alude a la preparación de la inteligencia y el carácter de los niños para que vivan en sociedad. Luego Moliner da un giro para especificar que se prepara a alguien para cierta función o para vivir en cierto ambiente o de cierta manera. Aquí se roza ya el hecho de que en medios y culturas diferentes se vive de maneras distintas, siendo la educación el medio para ayudar a los sujetos a acomodarse a las formas de ser y de actuar que definen lo cultural en su medio de pertenencia (Moliner, 2007: 102).

El historiador Georges Duby define la educación en el que califica como el sentido más amplio como "todas las comunicaciones entre el individuo y lo que le rodea (...) los medios por los cuales recibe los modelos culturales". (Duby, 2000: 59).

La educación, en su acepción más amplia, es el conjunto de fenómenos por los cuales el humano va asimilando prácticas culturales en las que se ve inmerso. Es a través de dichas prácticas que se convierte en sujeto, se liga a otros con los que se identifica y que posibilitan la convivencia entre ellos. Pero sabemos por experiencia que la convivencia entre humanos no es fácil, ya sea que pongamos la mira en lo micro-social, digamos en un salón de clases, o a nivel macro en las interacciones políticas a lo largo y ancho del mundo, encontramos de continuo conflictos que nos desbordan, que nos dejan perplejos, para los que, difícil y sólo, parcialmente encontramos soluciones.

Anthony Elliot, aludiendo a Freud, nos ayuda a comprender la complejidad que nos habita como sujetos:

Es gracias a la ambivalencia inconsciente, a la riqueza polisémica de la pasión humana, como Freud pudo elaborar una teoría de la subjetividad humana que nos la muestra frágil, escindida, múltiple, heterogénea. Una de las mayores contribuciones del psicoanálisis para reformular el concepto de individuo autónomo, que es una idea central de la cultura occidental moderna, reside en esta visión del sujeto como descentrado (...), rehabilitación psicoanalítica de la irracionalidad. (Elliot, 1998: 33).

\footnotetext{
${ }^{7}$ Sin más discusión.
} 
Nacidos en el desamparo, incapaces de sobrevivir por nosotros mismos durante nuestra prolongada infancia, surgimos como sujetos en una dependencia del amor de los otros que nos seguirá a lo largo de toda nuestra vida. Habremos de aprender a vivir luchando por gestar con esos otros, relaciones en las que prevalezca lo menos posible el sometimiento recíproco y en las que predomine la solicitud de asistencia, de atención, de cuidado mutuo en el respeto de las diferencias. Esto, que se dice rápidamente, es tarea de titanes que no cesa a lo largo de toda la vida. Necesitamos de los otros y a la vez hemos de combatir la tentación de controlarlos y de plegarnos a pautas de dominio que desdibujen nuestra subjetividad.

Reflexionando sobre la obra de arte, Walter Benjamin acuñó el concepto de "aura", a la que definió como "un especial entretejido de espacio y tiempo: una aparición única, una distancia que se preserva por más cercana que pueda estar" (Benjamin, 1991: 440). Y en otra parte comenta: "Este proceso es sintomático, su significación apunta más allá del ámbito artístico" (ibídem: 438). Y sí, diríamos que apunta a la subjetividad, lo que no es de extrañarnos, dado que son sujetos, humanos, los creadores de las obras de arte. Podríamos decir que el aura es aquello que nos distingue como sujetos únicos, irrepetibles, producto del misterio, incapaces de abarcarnos los unos a los otros; porque, no podemos ir más allá de nuestros contornos, los cuerpos nos separan y logramos, cuando más, empatizar, intentar ponernos en el lugar de ellos con sus propios enigmas, o dicho en alemán: 'einfühlen', sentir, así sea vagamente, el interior que los habita, el interior que nos habita, porque también para nosotros mismos somos en buena medida extranjeros, sujetos de un inconsciente inabarcable, y nuestro propio cuerpo es un cúmulo de mensajes, muchos de ellos indescifrables. Pulsamos, anhelamos, deseamos, tememos, nos angustiamos, preguntándonos a lo largo de toda nuestra vida quiénes somos, como se lo preguntaba una chica de doce años: "¿quién soy, siempre huyendo de mí misma, siempre a la búsqueda, siempre tratando de indagar qué sueños me habitan, que anhelos me alientan?

$\mathrm{El}$ actor educativo se acerca al sentir de los educandos y los alienta en su desarrollo, en la medida en la que escucha, palpa, presiente sus diferencias, sus singularidades y se desvirtúa, se aleja de su meta de promover su desenvolvimiento en la medida en que los iguala, los mercantiliza, los convierte en intercambiables, pretendiendo homogeneizarlos y someterlos a proyectos totalitarios, como lo fueron los que dieron lugar a tragedias de las más terribles que ha gestado la especie humana contra sus congéneres: el nazismo y el estalinismo.

\section{Identidad y cultura: cuestiones siempre abiertas}

¿Qué es aquello que nos hace percibirnos semejantes a otros, identificarnos con ellos y en ocasiones, así sea pasajeramente, sentir que vibramos al unísono con ellos como si estuviésemos hechos de la misma sustancia? Es un cúmulo de fenómenos anímicos, entre los que destaca la identificación, que está en la base de la gesta de las identidades, las cuales son en buena medida construcciones que se apoyan en lo social, en nuestras interacciones mediadas por oferta de roles y por instituciones. 
Educación, diferencias subjetivas y culturales. ¿De la multiculturalidad a la interculturalidad?

Nuestros deseos de estar de acuerdo con los otros, de coincidir con ellos, de encontrarnos y hasta de fundirnos amorosamente, son sólo uno de los momentos de la realidad de nuestros encuentros complejos e inevitablemente ambivalentes con ellos. En el otro extremo de nuestro anhelo de fusión está el de afirmarnos como diferentes, como sujetos deslindados aun de aquellos que más amamos. Buscamos ser acogidos, aceptados, amados, pero también distinguirnos y preservar nuestra autonomía. Y es en medio de ese ir y venir entre afectos disímiles, que gestamos, marcados por nuestras relaciones sociales, identidades: múltiples, complejas, en permanente transformación.

Las identidades que nos llevan a definirnos como profesionistas, como miembros de asociaciones, como portadores de un papel social: madres, padres, abuelas o abuelos, etcétera, las gestamos en las relaciones que establecemos con otros, en contextos específicos en los que se nos asignan ciertos roles, se nos califica con ciertas características, y donde asumimos en parte las designaciones; pero, también parcialmente, nos posicionamos críticamente ante ellas y las desafiamos.

En el acontecer cotidiano, en prácticas que muchas veces son repetitivas o son inducidas ritualmente, se favorece la asignación, respectivamente asunción de identidades. Con todo, donde quiera que los seres humanos entren en relación, interactúen, vivan situaciones conflictivas y negocien entre ellos, se darán procesos de construcción, respectivamente transformación de identidades. Por otra parte, las identidades se apuntalan en la comparación con los diferentes; por ejemplo, en situaciones grupales donde hay características que reúnen a ciertos sujetos en un subgrupo y a otros en uno alternativo. En el ámbito escolar, la división por grupos, grados o clases, es una de las formas más generalizadas de dar/asumir identidad, por ejemplo, los alumnos del primer grado, se diferencian claramente de los de sexto.

En torno a la pertenencia étnica o cultural se gestan también identidades, pero éstas no se basan en características o improntas cerradas, inamovibles, sino que están en permanente proceso de hibridación.

En su artículo Lo extranjero - tótem y tabú en el psicoanálisis, Mario Erdheim explica:

Las instituciones tienen con frecuencia el poder de generar la realidad virtual que confirma ilusoriamente sus teorías, y recíprocamente, uno tiende, como perteneciente a una institución, a pensar aquello que es parte de la ilusión institucional.

Y añade que es muy difícil conceptualizar y entender lo diferente, lo no coincidente al interior de las instituciones, porque hay una tendencia a combatir y excluir aquello que las desestabiliza o las pone en cuestión. Pero más adelante matiza:

Con todo, vale la pena pensar de nueva cuenta el concepto de cultura esta vez a partir de lo extranjero. En lugar de equiparar a la cultura con lo conocido, lo confiable, familiar desde tiempos inmemorables, 
podemos relacionarla con lo extranjero: cultura es desde este punto de vista lo que se origina en la confrontación con lo diferente, es en cierta medida el producto de la transformación de lo propio a través de la aceptación de lo extranjero. Y prosigue en un espíritu que nos hace pensar en el artículo de Freud (1919) Das Un-heimliche: Lo in-quietante: Si la cultura surge y se desarrolla ahí donde se da un encuentro con lo extranjero, entonces ella misma estará siempre impregnada de algo extraño, que a la vez que tiene un potencial generador de angustia lo tiene para producir fascinación. En la música y los cuadros de vanguardia se expresa con especial claridad este aspecto de la cultura" (Erdheim, 2000: 177-178).

Por su parte el teórico del poscolonialismo Homi Bhabha, define las culturas como estructuras formadoras de símbolos que están descentradas, esto es, que a través del desplazamiento de sus límites se abren a la posibilidad de articulación con lo diferente y están en un continuo proceso de hibridación. Hace una distinción entre los conceptos de "diversidad cultural” y "diferencia cultural" y dice que es la diferencia cultural la que "permite el proceso de enunciación de la cultura como 'susceptible de ser conocida', con autoridad, adecuada para la construcción de sistemas de identificación cultural." Hace ver cómo, cuando se habla de "diversidad cultural" se hace aparecer a las culturas como cúmulos de manifestaciones éticas, estéticas y etnológicas distintas, pero más o menos equivalentes y ubicadas a lo largo de un continuo, mientras que asumir las diferencias de cultura a cultura permite comprenderlas como procesos de significación que posibilitan la producción de campos de fuerza, de referencia, de aplicabilidad y capacidad (Bahbha, 2002: 207).

Lo que Bhabha busca hacernos ver, es que las culturas no son conjuntos de significados llanos $\mathrm{y}$ transparentes, sino que tienen zonas oscuras e inconscientes e implican intereses de grupo, y que se busca defender la forma de entender el mundo que ha resultado funcional para cada uno de esos grupos, por lo que el enfrentamiento de culturas implica lucha política y exige crear ámbitos de apertura, encuentro, comprensión y negociación; lo cito:

El pacto de interpretación nunca es simplemente un acto de comunicación entre el Yo y el Tú designados en el enunciado. La producción de significado requiere que estos dos lugares sean movilizados en el pasaje a través del Tercer Espacio, que representa tanto las condiciones generales de lenguaje como la implicación específica de la declaración en una estrategia preformativa institucional que no puede ser 'en sí misma' consciente. Lo que esta relación inconsciente introduce es una ambivalencia en el acto de la interpretación" (ibidem: 208).

En otras palabras, lograr acercamiento y entendimiento intercultural presupone un proceso de traducción y translación de códigos, de apertura a lo diferente, de elaboración; implica tolerancia a la ambigüedad y a la ambivalencia, enfrentamiento a lo polivalente, reconocimiento de que la interpretación que del mundo hace la propia cultura no es la única posible, y disposición a la generación de nuevos sentidos y a la movilidad social. 
Educación, diferencias subjetivas y culturales. ¿De la multiculturalidad a la interculturalidad?

El concepto de "diferencia cultural" diluye el mito de las culturas como códigos integrados, en desarrollo progresivo continuo, en expansión, y nos invita a reconocer la permanente reorganización no lineal, sino dialéctica, de nuestras tramas de sentido, que como órdenes simbólicos son parte de un círculo generativo que parte de necesidades existenciales y por ende se transforma en relación con los cambios en las condiciones de vida. Reconocer las diferencias implica también percatarnos de la temporalidad discontinua y la intertextualidad que afecta a todos los mundos simbólicos actualmente vigentes. Aceptar las limitantes de nuestras cosmovisiones, puede, como asegura el mismo Bhabha, "abrir el camino a la conceptualización de una cultura internacional basada no en lo exótico o en el multiculturalismo de la diversidad de las culturas, sino en la inscripción y articulación de la hibridez cultural" (ibídem: 209).

\section{La tentación de destruir lo extranjero}

A lo largo de la historia existen testimonios de actitudes diferentes, incluso en apariencia diametralmente distintas en relación a los extranjeros.

En la antigua Atenas, la hospitalidad para con el extranjero era considerada una obligación. El Edipo en Colono de Sófocles, nos muestra al rey Teseo que acude al llamado de acogimiento del trágico héroe, como la encarnación de virtudes atenienses, ante todo la lealtad y la hospitalidad. El siguiente pasaje de la citada tragedia deja ver aspectos de la historia de Teseo que fueron fundantes de su actitud humanitaria:

\section{“(Entra Teseo con su escolta.)}

TESEO. - Te he reconocido, oh hijo de Layo, por haber oído a muchos hablar hace tiempo de la sangrienta destrucción de tus ojos. Ahora, por lo que he escuchado en mi camino hacia aquí, tengo ya la certeza. Pues tu aspecto y tu lamentable rostro nos evidencian que eres quien eres, y tras compadecerte quiero preguntarte, desventurado Edipo, con qué ruego para la ciudad y para mí, tú en persona y tu infeliz acompañante, os habéis presentado. Indicádmelo. Pues un terrible suceso tendrías que comunicarme para que yo me desatendiera, cuando sé que yo mismo, como tú, fui educado en el destierro y que más que cualquier hombre arrostré en tierra extranjera peligros con riesgo de mi propia persona, de modo que a nadie que sea extranjero, como tú ahora, dejaría de ayudar a salvarse. Sé que soy mortal y que en nada dispongo más que tú del día de mañana" (Sófocles, 1992: 534).

Más adelante, Edipo da cuenta de la transformación que pueden sufrir las relaciones entre países pudiendo afectar la disposición a la hospitalidad, pero luego Teseo reasegura la firmeza de su actitud de acogimiento:

TESEO. - ¿Qué desgracia temen que les venga de los oráculos? EDIPO. - Que sea inevitable el ser derrotados por este país. 


\section{Emma Ruiz Martín del Campo}

TESEO. - ¿Y cómo podrían llegar a estar tirantes nuestras relaciones?

EDIPO. - ¡Oh queridísimo hijo de Egeo! La vejez y la muerte a su tiempo sólo a los dioses no alcanza. El tiempo, que todo lo puede, arrasa todas las demás cosas. Se consume el vigor de la tierra, se consume el del cuerpo, perece la confianza, se origina la desconfianza y no permanece el mismo espíritu ni entre los hombres amigos ni entre una ciudad y otra.

Para unos, pronto, para otros, más tarde, los placeres se vuelven amargos y, posteriormente, dulces. Asimismo, si a Tebas por ahora le va bien en sus relaciones contigo, el tiempo incalculable en su curso engendra días y noches sin cuento durante los cuales se pueden romper por la lanza, con un pequeño motivo, los amistosos acuerdos de hoy. Entonces mi cadáver en reposo, enterrado, beberá, ya frío, la caliente sangre de ellos, si es que Zeus es aún Zeus y Febo hijo de Zeus es infalible.

Pero no es lícito hablar de asuntos que deben ser inviolables. Déjame, pues, en el punto en que comencé: que guardes sólo tu juramento, y nunca tendrás que decir que recibiste en Edipo a un inútil habitante de estos lugares, si es que los dioses no me engañan (...)

TESEO. - ¿Quién es el que, en esta situación, rechazaría el favor de un hombre así con quien, en primer lugar, existe siempre un hogar común entre nosotros por los vínculos de hospitalidad ${ }^{8}$ y luego, tras venir como suplicante de los dioses, satisface un tributo no pequeño para esta tierra y para mí? Yo, temeroso ante esto, nunca desdeñaré su ofrecimiento y le instalaré en esta región como ciudadano" (Sófocles, 1992: 535- 536).

Por otra parte, en el Diccionario manual de las supersticiones alemanas editado por Meyer (1974), aparece lo siguiente ligado al término Fremder (extranjeros):

En general se le enfrenta con gran desconfianza... No se le muestra a un recién nacido, ni a los niños pequeños, en especial a los no bautizados. Si él quiere ver al niño, ha de ponerle él al pequeño agua bendita o decir 'que dios lo proteja', para subrayar sus buenas intenciones y paralizar su influencia maligna...

A ningún extranjero le está permitido mirar en el cubo de la leche, ni estar presente durante la ordeña, Seihen, o producción de mantequilla; de preferencia no se le permite entrar al establo... si entra, ha de decir al ganado que dios lo proteja, o 'mucha suerte en el establo' (...) Los extranjeros son presagio de peste, de guerra, hacen temer la posibilidad de una aparición demoniaca, por eso no se han de recibir demasiado pronto regalos de ellos".

Para Erdheim, "la esencia del concepto de extranjero es lo que está fuera de la cultura9: los pueblos que viven en contacto directo con la naturaleza, los salvajes, los locos. No se experimenta con ellos punto alguno en común a partir del cual se pudiere aprender a transformar lo propio. La relación

\footnotetext{
${ }^{8}$ Negritas de la autora.

${ }^{9}$ De una cultura determinada.

${ }^{10}$ Piensan los que desean preservar el estado de cosas a toda costa.
} 
Educación, diferencias subjetivas y culturales. ¿De la multiculturalidad a la interculturalidad?

con el extranjero es en primera línea una relación de poder y de defensa. De los extranjeros sólo puede esperarse destrucción ${ }^{10}$, por tanto hay que proteger de ellos lo que nos pertenece. Freud habla en este contexto de la 'animadversión latente (a la cultura) de las capas sociales más favorecidas', que no se ha percibido suficientemente. Quizá sería mejor hablar de 'animadversión ante la historia' de las capas sociales que tienen las mejores condiciones de bienestar. En tal caso quedaría claro que es de la transformación de la cultura de lo que ellas se defienden. Para ellas lo extranjero que no pueden integrar a su historia queda escindido y de ser posible lo destruirían" (Erdheim, 1991: 250-251).

Desde el punto de vista de la subjetividad, la forma de enfrentar lo extranjero tendrá que ver con tendencias que nos abarcan a todos, pero que se actualizan de formas muy diversas, de acuerdo a las distintas historias de vida y experiencias tempranas y actuales de los sujetos.

Aquello de nosotros que se ha ido al exilio de nuestro inconsciente, puede llegar a ser proyectado en el ajeno, el desconocido, el extranjero, en quien recaen fácilmente nuestras ambivalencias.

A lo largo de nuestra socialización aprendemos y compartimos con nuestros prójimos próximos, formas de entender el mundo, de reaccionar frente al amor, la muerte, lo considerado sagrado y lo juzgado intolerable, los valores éticos y estéticos, etcétera. Recibir al extranjero y convivir con él confronta, y puede poner en cuestión esas formas aprendidas, así como remover lo que, censurado en nuestra cultura y/o subcultura, a nivel micro o macro-social, quedó 'atrapado' en el más allá de nuestra consciencia, en nuestro extranjero interno, nuestra otredad. Es pues, difícil, aceptar a los otros, tanto con sus aspectos diferentes a nosotros, como con aquellos en que somos semejantes, de ahí que el encuentro entre sujetos procedentes de culturas distintas, es un reto que podemos enfrentar con la esperanza de lograr integraciones creativas, pero que da lugar también a cuestionamientos, hechos desde la variedad de múltiples disciplinas, relativos a la diferencia, a lo que da lugar a ella, y luego, cuando es el caso, a la hibridez lograda y la interculturalidad.

\section{Culturas, sujetos, experiencia y creatividad}

Iniciemos este apartado cuestionándonos qué es ese Otro cultural. Las culturas son tramas simbólicas a las que nos articulamos los sujetos, tramas simbólicas que, por cierto, están en un proceso de permanente evolución e hibridización y que nos posibilitan encontrar sentidos en medio del caos y la entropía que amenazan siempre con acometer y dar cuenta de nuestra vulnerabilidad.

La pertenencia cultural, sin embargo, tiene también un costo para el sujeto, al que se le exige renunciar a muchas satisfacciones para adecuarse a la comunidad. Hay una permanente dialéctica que pone a los sujetos frente a las prácticas culturales. Los sujetos del deseo asumimos-repelemos las exigencias e imposiciones del Otro cultural que se manifiesta a través de las instituciones, entre ellas las educativas. Las fantasías y los deseos se entretejen profundamente en las actitudes que tomamos frente a la ley y el orden social. Y por otra parte, las prácticas educativas de las diferentes culturas y comunidades que en ella se acogen, varían, y si bien algunas son subjetivantes, hay otras 
cuyo efecto atenta contra la subjetividad (pensemos en las exigencias de regímenes totalitarios, el adoctrinamiento de la juventud durante el nacionalsocialismo en Alemania, los mandatos que se imponen a los sujetos en las sectas fundamentalistas, que llegan en ocasiones a exigirles el suicidio, etcétera).

Y es que educar, socializar, implica, como ya hemos visto, sujetar a tramas simbólicas diferentes y en formas complejas que presentan ciertas variaciones y que dejan al sujeto un grado menor o mayor de libertad en la configuración de su vivir (que por otra parte está también marcado por el azar). La renuncia a la descarga espontánea de impulsos y búsqueda de satisfacción, que inevitablemente implica el proceso de subjetivación, ocurre en cierta medida por amor a los otros: cuidadores, gestores, pero también y muchas veces sobre todo, por temor al rechazo, al desprecio, al desamparo, al caos y en última instancia a la muerte. La dialéctica sujeto/sociedad es compleja e implica la búsqueda de un difícil equilibrio entre, en un extremo la sumisión al amo, la masificación, la obediencia ciega a postulados planteados como irrenunciables o la confrontación destructiva y aniquilante con toda forma de institucionalización. En cuanto a las propuestas contenidas en diversos marcos de socialización, afirma Javier Meza: "Las obsesiones de los que gobiernan guiados por el pensamiento occidental sólo cambian ligeramente: antes, a su juicio, todos debíamos ser santos, para otros, todos proletarios, ahora, todos comerciantes, o más bien "santos comerciantes" (Meza, 2008: 81).

Con todo, en los permanentes ajustes de los sujetos con las culturas que habitan, juega también un papel importante el potencial con el que tales sujetos cuentan de llegar a transformar algunos elementos de esa(s) cultura(s) en que están insertos. Las tramas simbólicas que dan cuerpo a lo cultural no son estructuras fijas que nos determinan en forma total e inalienable. Son más bien, como ya dijimos, entretejidos simbólicos que están en continua renovación. De ello da cuenta el cambio cultural en el que también participan los sujetos.

De ahí que nos preguntemos: ¿dónde se gesta el potencial instituyente de tales sujetos? Podríamos decir que proviene de la posibilidad que tienen de hacer saltar los límites de los significantes para generar nuevos discursos, a partir de la experiencia, de la expresión de la singularidad a través de la fantasía. Los sujetos podrán innovar en tanto se hayan liberado de instituciones que se han vuelto escleróticas, anquilosadas.

Los sujetos, inmersos en la atmósfera de instituciones educativas de su cultura, se enfrentan al reto de no vivir tales instituciones como incuestionables, asumiendo acríticamente sus exigencias. La experiencia, el diálogo y la escucha, el acercamiento al otro que nos habita y da cuenta de nuestros deseos más profundos, son vías para la emergencia de la creatividad, de la innovación, de la transformación cultural. Al respecto Gárate y Marinas comentan:

Frente a la cultura del bienestar, difundida por una ciencia oficial que estratifica los conocimientos y distribuye etiquetas de novedoso o de caduco, según l'air du temps', sería indispensable ahondar, volver de 
Educación, diferencias subjetivas y culturales. ¿De la multiculturalidad a la interculturalidad?

nuevo a los restos del malestar en la cultura y recoger, sin estratificaciones ideológicas o instrumentales, los fragmentos que por no hallar cabida en el discurso universitario se refugian en los saberes de experiencia. Todo lo que no cabe en los límites de la sanción oficial, todo lo que no forma parte de 'lo santo', lo proclaman, por los resquicios y en los umbrales, las voces que rebosan y se escapan: es el efecto subversivo de la experiencia (Gárate y Marinas, 2003: 62).

El cambio cultural significativo y el camino exploratorio e imaginativo que conduce a él, puede ser ejemplificado con la experiencia creativa de Freud en el descubrimiento del inconsciente. La del médico vienés resultó ser una verdadera experiencia, una ruta innovadora para explorar la subjetividad, que se hizo posible desmontando prejuicios, inaugurando la escucha, que ya no la mirada y la auscultación, como medios para acercarse a los sujetos, a sus síntomas, a sus mensajes cifrados, a su deseo. Ante la exigencia de una de sus pacientes, de que no le indicase una ruta específica al hablar, Freud apeló a la llamada "asociación libre".

En dicha "asociación libre", al decir de Claudia Weiner: "Se apuesta en cada ocurrencia a una singularidad que haga saltar la estructura que permite movimientos de agujeros, cortes, pliegues y transformaciones, a saber, la estructura del lenguaje”. Y luego se pregunta: “¿De qué se trata en el lenguaje?

Un sueño, un dolor, la risa o el llanto, lo imaginado, lo experimentado, una partitura, la letra de un poema..." Después su discurso deriva hasta llegar a enunciar el potencial creativo, instituyente de la ficción (la imaginación, la fantasía preñada de contenidos inconscientes): "La ficción no confirma la realidad, la produce. El atractivo de este género no es imaginar mundos que no existen, sino hacerlos existir" (Weiner, 2009: 121).

Enunciemos en otros términos esta cuestión central que nos ocupará más abajo: ¿cómo educar y ser educados sin que se anule nuestra creatividad, nuestro potencial instituyente, que es condición de la experiencia innovadora y por tanto de posible cambio subjetivo y cultural? Tal cuestión, por otra parte, hay que referirla a la tarea cultural llevada a cabo en nuestras sociedades del siglo XXI, preñadas de racionalismo técnico y de un mercantilismo ligado, entre otras cosas, a la creciente globalización.

Los riesgos y carencias que nos afectan a los sujetos que habitamos la Tierra en este siglo XXI (cambio climático, tasas elevadas de desempleo, creciente desigualdad entre comunidades ricas y pobres, etcétera), pueden, según nos hace ver Elliot, "ser experimentadas como extremadamente amenazadoras y abrumadoras", por lo que podrían dar lugar a "defensas rígidas contra la intensidad de la experiencia humana" (Elliot, 1998: 36).

A lo que Elliot alude en la anterior cita, es a la tentación de algunos humanos de plegarse excesivamente al control, al orden institucional, al dominio, renunciando al potencial crítico e innovador, ante una realidad que parece estrechar las perspectivas de futuros habitables para la especie humana. 
Una tarea de la educación, entre otras, sería impedir que tales defensas surgieran, crecieran, se estratificaran en los educandos, a fin de que se diera la apertura a los otros y al Otro de una manera crítica y reflexiva. Educando se tendería a la gesta de sujetos que puedan experimentar, innovar, que den lugar a una creatividad que no quede cautiva en estereotipos mercadotécnicos o tecnocráticos, ni tenga la esclerosis de las instituciones totalitarias y/o fundamentalistas. Se trata de liberar la palabra, la fantasía, de eliminar etiquetas, abrirse a la escucha, a la singularidad, a la gesta de proyectos innovadores, aunque no sean de la envergadura de los sueños caídos de progreso permanente ni de las grandes utopías. Sería bastante educar para la vida, para tolerar las angustias y ambivalencias, la incertidumbre, los límites propios y los de los otros sin renunciar a nuestro potencial deseante e instituyente. Sería bastante con contribuir al despertar de sujetos ávidos de plantear nuevas preguntas y rastrear respuestas para los acuciantes problemas que nos aquejan a los humanos, a otras especies, al planeta en esta agitada posmodernidad.

\section{Los sujetos de la educación en un mundo cada vez más globalizado}

Las culturas "premodernas" se sustentaban en rituales colectivos y en tradiciones y formas de vida "recicladas" en una territorialidad más o menos circunscrita. Los sujetos crecían preparados para ocupar un lugar definido en su comunidad, marcado por roles sociales. Desarrollaban un fuerte sentido de pertenencia y se identificaban con los demás miembros de su cultura, considerando en buena medida a los de fuera, los extranjeros, como potenciales enemigos o bien normando con reglas lo más claras posibles la hospitalidad.

Las culturas posmodernas se caracterizan por la acentuada hibridación en un contexto global tecnificado. No se cohesionan en torno a discursos incuestionables. Los sujetos son aparentemente más libres, pero también vulnerables psicológicamente, en tanto se reconocen expuestos a un sinnúmero de riesgos que les son conocidos no sólo por efecto de la comunicación directa persona a persona, sino a través de las tecnologías de la información. Como argumenta Elliot: "El avance de la modernización tecnoeconómica se relaciona cada vez más con la producción de riesgos, peligros e inseguridades en una escala global sin precedentes" (Elliot, 1998: 101). Y ¿qué caracteriza a la globalización del siglo XXI, de la posmodernidad?

Nos referimos a la globalización como un conjunto de procesos de intercambio mundial que desembocan en nuevas formas, escalas y perspectivas de contactos sociales, económicos y culturales. Dichos intercambios se dan entre los más diversos actores sociales. Si la globalización es un proceso que se inició hace ya varios siglos con la exploración de los mares, la concepción de la tierra como un todo que incluía diversos continentes, etcétera, en la actualidad se habla de este fenómeno con especial insistencia debido a que los intercambios de sujetos, de información, de comercio, etcétera, han adquirido unas proporciones y una rapidez antes inimaginables. El desarrollo de los medios de comunicación, el avance de las tecnologías y las migraciones a gran escala, el mercado convertido en eje de las relaciones internacionales, entre otras cosas, han acelerado el 
Educación, diferencias subjetivas y culturales. ¿De la multiculturalidad a la interculturalidad?

cambio histórico, han reducido la soberanía de los Estados Nacionales, han producido profundos cambios en las culturas antes vistas como locales y con cierta estabilidad.

La profunda redefinición de la escena internacional ha incidido también en las formas de subjetividad prevalecientes y en el fenómeno educativo, de ahí que nos preguntaremos por algunas de las implicaciones y transformaciones perceptibles en los sujetos y en el campo educativo en la era de la globalización incrementada, de la llamada posmodernidad.

El mundo posmoderno nos pone como sujetos ante nuevos retos, ante modalidades inéditas de encuentro con nosotros mismos y con nuestros congéneres. Nos vemos inmersos en la paradoja de la posibilidad de acercamiento virtual de unos con otros en cuestión de segundos y desde los rincones del mundo más distantes entre sí, mientras que por otra parte la desintegración de espacios comunitarios de contención nos hace estar más solos que nunca en la tarea de planificar nuestras vidas y encontrar un sentido para las mismas. La información a la que actualmente tenemos acceso a través de los medios masivos de comunicación y las nuevas tecnologías, puede ser avasalladora. La oferta de modelos de identificación es más variada que nunca. Ante la incertidumbre de los panoramas presentes y futuros tanto a nivel local como global (pobreza y desempleo en aumento, violencia y riesgos de ingobernabilidad, crisis energética, calentamiento global, etcétera), los sujetos experimentamos con mayor intensidad que en otras épocas la discontinuidad, la diferencia. Nos vemos empujados a una permanente reconstrucción de nosotros mismos y de nuestros mundos. Al respecto dice Elliot: "La subjetividad posmoderna está constituida a través de un volverse de la fantasía sobre sí misma con el fin de hacerse cargo de la ambivalencia e incertidumbre de entendimiento y mundo. Esto significa proceder sin pautas absolutas ni autoridad definitiva en la vida personal y cultural, tolerando la incertidumbre y la confusión e intentando pensar lo impensable dentro de la turbulencia de los procesos sociales contemporáneos” (Elliot, 1998: 16).

En nuestro mundo de apertura global de las fronteras propio / otro, prevalece un consumismo pasivo y una entrega no cuestionada a la técnica Por otra parte, mercado y técnica son actualmente elementos centrales de los procesos de subjetivación, y ¿qué clase de sujetos generan? Unos que creen poder comprar el placer, la serenidad, la paz interior, eliminar conflictos. Cuando en realidad no se puede prescindir de los otros como objetos del deseo, de ahí la proliferación de malestares relacionados con el aislamiento, la impotencia de sujetos más o menos solitarios que muchas veces pretenden llenar con productos o con sensaciones la carencia de relaciones amorosas y espacios que permitan dar lugar al vínculo social.

Sin embargo, si el predominio del mercado y la disolución o el debilitamiento de marcos tradicionales de sentido han tenido por un lado un precio psicológico muy alto: la equiparación de muchos intercambios en la relación humana a intercambios mercantiles, o el ensimismamiento de los sujetos, entre otras cosas, la vuelta de los sujetos hacia sí mismos podría ser utilizada creativamente en el campo educativo. Ello en tanto los educadores estuvieren abiertos a la escucha y a la 
actividad de la imaginación inconsciente. Se trataría de pasar de centrarse en sí mismos predominantemente dirigiendo la mirada hacia el propio interior, al descubrimiento de sí mismo y de los otros a partir del lazo social y de los efectos que con nuestras interacciones producimos en otros, tanto como los que ellos generan en nuestra subjetividad.

Sería deseable una educación que dé lugar a una actitud de sujetos deseantes que no se dejan apabullar en su deseo, que aceptan su finitud sin convertirse en presas del desaliento y la sumisión y que buscan, a pesar de la aridez del panorama, formas creativas para enfrentar la crisis del planeta y de la humanidad.

\section{A manera de conclusión}

$\mathrm{Al}$ abordar la cuestión de las diferencias culturales y las dificultades que se presentan en ambientes educativos en los que coinciden sujetos procedentes de etnias y /o medios de socialización distintos, me parece importante resaltar el hecho de que todos, de diversas maneras y en distintos momentos experimentamos sensaciones de extrañeza, de ser extranjeros en ciertos contextos sociales y en determinados momentos existenciales. Podemos entonces preguntarnos cómo podríamos aprovechar tal experiencia en los encuentros con los diferentes.

Una cuestión fundamental es indagar si los otros diferentes con los que nos encontramos, manejan el lenguaje en el que estamos hablando, de no ser así hay que tender puentes de comunicación a través de traductores, de apertura al uso de objetos simbólicos y lenguaje no verbal en la comunicación, o utilizar expresiones artísticas, para abrir ámbitos de acercamiento, que les permita sentirse aceptados en su singularidad en el medio para ellos desconocido. Simultáneamente, si los alumnos son migrantes que llegan para quedarse, una medida necesaria, cuando su situación emocional se los permita, es que los alumnos se integren en clases del idioma de uso generalizado en la comunidad y/o en la institución educativa que los acoge.

Afanarse en que los estudiantes adquieran conocimientos sin que ellos estén en la disposición afectiva mínima para hacerlo es inútil. Se requiere calma interior, contención de sí mismo, garantía de contar con interlocutores, docentes en el caso del que nos ocupamos, interesados no sólo en el rendimiento, sino en atender a su singularidad. Se trata de llegar al punto en que, ganando confianza en el nuevo medio se despierte su curiosidad, su motivación, su interés para buscar caminos de inserción en él, uno de los cuales podría ser los conocimientos que se ponen a su alcance.

Tomando en cuenta que la migración es en muchos casos un evento traumático que implica desprendimientos dolorosos y en ocasiones vivencias de violencia y crueldad, las escuelas que acogen a un gran número de alumnos de diferentes culturas habrían de implementar nichos dedicados a que ellos puedan hablar de sus experiencias y compartirlas con otros que las escuchen con interés. Tales nichos podrían ser espacios psicoterapéuticos cuando se cuenta con los profesionales para el caso, pero cuando no los haya a la mano, se podrían abrir grupos dirigidos por facilitadores que por su entrenamiento y motivación de dar apoyo a los extranjeros, tengan la flexibilidad cultural, 
Educación, diferencias subjetivas y culturales. ¿De la multiculturalidad a la interculturalidad?

la apertura requerida para potenciar la gesta de esos espacios intermedios de los que hablan desde diferentes perspectivas Winnicott (1972) \& Bhabha (2002) en los que los inmigrantes puedan recuperar confianza y se facilite la gesta de hibridez cultural.

Apelando al diálogo cuando el nivel de manejo del idioma lo permita y al inicio a cantos, juegos, teatro, expresiones simbólicas, se pueden escenificar normas que rigen la vida tanto en el ambiente que dejaron los migrantes como en la sociedad a la que llegan. Tener la posibilidad de comparar, indagar, reflexionar y jugar las diferencias culturales es un camino para la apertura de espacios intermedios de gesta de hibridez cultural.

Los auxiliares en el camino de recepción de los alumnos de culturas diferentes a la del ambiente escolar han de tener información sobre dichas culturas, adquirida en el mejor de los casos a través de la pasión y el deseo de comprender cosmovisiones y mundos diferentes, y más importante todavía, han de tener el profundo deseo de entender singular y sensiblemente a los otros, a la búsqueda de poner medios para un posible encuentro con ellos.

Condición sine qua non de la gesta de hibridez cultural es, como lo plasma el ejemplo de Khoumba, que haya respeto mutuo, que los miembros de todas las minorías representadas en el salón de clase se sientan acogidos, que no sean amenazados ni discriminados en función de sus creencias, sus tradiciones, los usos y costumbres en los que fueron socializados en los mundos de los que proceden. La discriminación produce movimientos de retirada y defensa y frena la gesta de espacios potenciales para la gesta de la interculturalidad.

Por otra parte, los docentes en estos grupos multiculturales, sabemos, se enfrentan a una labor de una alta complejidad y habrían de contar a su vez con los apoyos personales e institucionales necesarios para llevar a cabo su tarea.

Terminaré apuntando a una difícil realidad: la integración de migrantes en los países a los que llegan y de alumnos en el medio escolar, es un problema de múltiples vertientes. No bastan los apoyos que puedan brindarse a los sujetos para elaborar anímicamente las experiencias vividas en la migración. Se requiere también que la política proteja y conceda derechos a las minorías étnicas y culturales, a fin de que los sujetos que las integran ocupen un lugar social que les permita integrarse creativamente al quehacer cultural. Ésta, sin embargo, es una cuestión que no es competencia directa de los docentes y que excede los límites de reflexión planteados en este trabajo.

Como profesionales de la educación, sensibles a las diferencias culturales, lo fundamental es entregarnos a la práctica docente con la apertura y tolerancia necesarias, para impulsar la empatía y la mayor comprensión posible entre los integrantes de los grupos a nuestro cargo. Se trata de generar una atmósfera creativa, receptiva a la innovación requerida en un mundo que se transforma a un ritmo cada vez más acelerado y en el que la movilidad de los sujetos a lo largo y ancho del mundo y la comunicación entre culturas distintas se da a niveles en otras épocas inimaginables. 


\section{Bibliografía}

Bégadeau, F. (2007). Entre les murs. París: Galimard.

Benjamin, W. (1991). Das Kunstwerk im Zeitalter seiner technischen Reproduzierbarkeit (La obra de arte en la era de su reproductibilidad técnica). En Benjamin Walter: Abhandlungen. Gesammelte Schriften, Band I-2 (pp. 435-508). Frankfurt, Suhrkamp Taschenbuch Wissenschaft. Bhabha, H. (1994). The Location of culture, London and New York: Routledge.

— (2002). Cultural Diversity and Cultural Differences (Diversidad Cultural y Diferencias Culturales). En Ashcroft, B; Griffiths, G and Tiffin, H. The Post-colonial Studies Reader (El Lector de Estudios Postcoloniales) (pp. 206-210). London and New York, Routledge.

Derrida, J. (2000). De la Gramatología. México: Siglo XXI Editores.

Duby, G. (2000). Historia de las Mentalidades. En Rojas, Beatriz. Obras selectas. México. Fondo de Cultura Económica.

Elliot, A. (1998). Sujetos a nuestro propio y múltiple ser. Buenos Aires: Amorrortu.

Erdheim, M. (1991). Die Repräsentanz des Fremden” (La representación de lo extranjero). En Psychoanalyse und Unbewusstheit in der Kultur (Psicoanálisis e Inconsciencia en la Cultura) (pp. 237-251). Frankfurt: Suhrkamp-Taschenbuch-Wissenschaft.

— (2000). Das Fremde-Totem und Tabu in der Psychoanalyse (Lo extranjero-Tótem y Tabú en el Psicoanálisis). En Streeck, Ulrich (Hg.) Das Fremde in der Psychoanalyse (Lo extranjero en el psicoanálisis). Giessen: Psychozosial-Verlag.

Forst, R. (2003). Toleranz im Konflikt. (Tolerancia ante el conflicto). Frankfurt: Suhrkamp.

Freud, S. (1919). Das Unheimliche. (Lo In-quietante). Frankfurt: Fischer Taschenbuch Verlag, Studienausgabe, Band IV: 241-274.

_ (1933). Neue Folge der Vorlesungen zur Einführung in die Psychoanalyse (Nueva Serie de Lecciones Introductorias al Psicoanálisis). Frankfurt: Studienausgabe, Editorial Fischer.

Gárate, I. \& Marinas, J. M. (2003). Lacan en Español. Madrid: Biblioteca Nueva.

Geertz, C. (2001). La Interpretación de las Culturas. Barcelona: Gedisa.

Gstettner, P. (1999). Subjektivität im interkulturellen Lernprozess (La subjetividad en el proceso de aprendizaje intercultural). En Aluffi-Pentini, A; Gstettner, P.; Lorenz, W; Wakounig, V (Hrsg.). Antirassistische Pädagogik in Europa. Theorie und Praxis (pp. 183-200). Klagenfurt: Drava Verlag.

Meyer (1974). Handwörterbuch des deutschen Aberglaubens (Diccionario manual de las supersticiones alemanas). Waage Zypresse: Nachträge.

Meza, J. (2008). La pastoral del miedo fraguado en la culpa. En Tramas, (pp. 59-92) Núm. 30. México: Revista de la Universidad Autónoma Metropolitana, Unidad Xochimilco.

Moliner, M. (2007). Diccionario de uso del español. Madrid: Gredos.

Orgris, S. (2006) Minderheiten -Leben und überleben in einer globalisierten Welt. Identitäten, Anpassung und Widerstand (Minorías - vida y supervivencia en un mundo globalizado. Iden- 
tidades, individualidad, emancipación). En Ethnopsychoanalyse 7: Solidarität, Individualität, Emanzipation (pp. 96-132). Frankfurt: Brandes \& Apsel.

Sófocles (1992). Edipo en Colono. En Tragedias (pp. 499-578). Madrid: Biblioteca Clásica Gredos. Wakounig, V. (1999). Verstrickt in den eigenen Rassismus: Minderheiten in einer anderen Rolle (Enredadas en sus propios racismos: Minorías jugando un papel distinto). En Aluffi-Pentini, A; Gstettner, P.; Lorenz, W; Wakounig, V (Hrsg.). Antirassistische Pädagogik in Europa. Theorie und Praxis (pp. 148-159). Klagenfurt: Drava Verlag.

Weiner, C. (2009). La Promesa Preformativa de la Ciencia Ficción. En Artefactos (pp. 116-130). Revista de la elp, núm. 1. México.

Winnicott, D. (1972). Realidad y Juego. Buenos Aires: Gedisa.

Recibido: 23/09/13

Dictaminado: 25/02/14

Corregido: 02/03/14

Aceptado: 03/03/14 